\title{
Is the Sun a Magnet?
}

\author{
D.O. Gough ${ }^{1,2,3}$ (iD
}

Received: 11 November 2016 / Accepted: 27 March 2017 / Published online: 19 April 2017

(C) The Author(s) 2017. This article is published with open access at Springerlink.com

\begin{abstract}
It has been argued (Gough and McIntyre in Nature 394, 755, 1998) that the only way for the radiative interior of the Sun to be rotating uniformly in the face of the differentially rotating convection zone is for it to be pervaded by a large-scale magnetic field, a field which is responsible also for the thinness of the tachocline. It is most likely that this field is the predominantly dipolar residual component of a tangled primordial field that was present in the interstellar medium from which the Sun condensed (Braithwaite and Spruit in Nature 431, 819, 2004), and that advection by the meridional flow in the tachocline has caused the dipole axis to be inclined from the axis of rotation by about $60^{\circ}$ (Gough in Geophys. Astrophys. Fluid Dyn., 106, 429, 2012). It is suggested here that, notwithstanding its turbulent passage through the convection zone, a vestige of that field is transmitted by the solar wind to Earth, where it modulates the geomagnetic field in a periodic way. The field variation reflects the inner rotation of the Sun, and, unlike turbulent-dynamo-generated fields, must maintain phase. I report here a new look at an earlier analysis of the geomagnetic field by Svalgaard and Wilcox (Solar Phys. 41, 461, 1975), which reveals evidence for appropriate phase coherence, thereby adding support to the tachocline theory.
\end{abstract}

Keywords Helioseismology, heliophysics $\cdot$ Solar magnetic rotator, geomagnetism

\section{Introduction}

In the original discussion of the dynamics of a tachocline separating a magnetically rigid solar radiative interior from the differentially rotating convective envelope, for illustrative purposes the simplest configuration was adopted: it was presumed that the axis of a dipolar

\section{D.O. Gough}

douglas@ast.cam.ac.uk

1 Institute of Astronomy, Madingley Road, Cambridge, CB3 OHA, UK

2 Department of Applied Mathematics and Theoretical Physics, Centre for Mathematical Sciences, Wilberforce Road, Cambridge, CB3 0WA, UK

3 Hansen Experimental Physics Laboratory, Stanford University, Stanford, CA 94305, USA 
interior magnetic field is aligned with the axis of rotation (Gough and McIntyre, 1998). Latitudinal differential rotation of the convection zone, established by the angular-momentum transport balance between Reynolds stresses, meridional circulation, and, possibly, Maxwell stresses, is hardly affected by the interior; it simply provides an upper boundary condition for the tachocline: a thin layer of transition from the rigidly rotating interior, whose rotation rate $\Omega_{0} / 2 \pi$ lies between the equatorial $(\simeq 460 \mathrm{nHz})$ and the polar $(\simeq 320 \mathrm{nHz})$ values in the convection zone. The interior rotation is controlled by the stress balance and angular momentum transport in the tachocline, and, of course, by the large-scale dipole magnetic field. Its rate has been established by helioseismology to be about $436 \pm 5 \mathrm{nHz}$ sidereal (Schou et al., 1998; Komm et al., 2003). Pressure imbalance brought about by the shear induces a meridional flow in the tachocline that is downwelling in both polar and equatorial regions. That flow advects the field against its natural tendency to diffuse outwards, largely confining it to the radiative zone. The descending meridional flow is deflected horizontally by the combined action of the stable stratification and the magnetic field, towards the equator in the polar regions and towards the poles in the equatorial regions. The motion converges in the vicinity of zero tachocline shear, near latitude $30^{\circ}$, where it is expected to be deflected upwards back into the convection zone, dragging with it some of the field, which is then distorted and amplified by magnetohydrodynamical processes commonly called dynamo action. The basic dynamics is dominated by the rotational shear and the pressure imbalance that it produces; the precise geometry of the magnetic field deep in the radiative interior has little direct influence on the details of the flow geometry in the tachocline, aside from its role of maintaining interior rigidity.

\section{Consequence of the Tachocline Flow}

Although usefully illustrative, an interior dipole magnetic field whose axis is aligned with the axis of rotation is unlikely to be stable ( $c f$. Gough, 2012), for the interior magnetic field structure is susceptible to further advection by the tachocline flow. In a strictly axisymmetric state, the associated net torque is null. However, because a dipolar field is most intense on and near its axis of symmetry, the equatorward horizontal tachocline flow near the rotation axis causes any slight deflection of the magnetic axis to be augmented: the magnetic axis of an initially aligned magnetic field would therefore migrate equatorwards until it meets the poleward flow from the equator, where it would come to rest, near latitude $30^{\circ}$. Similarly, if the axis of the field were initially lying in or near the equatorial plane, it would be advected polewards. The adjustment would not actually be as simple as I have sketched, and would involve distortion of the dipolar configuration and the consequent generation of Alfvén and magnetosonic waves. Moreover, it should be appreciated that I have described the development of the instability as envisaged in the frame of the convection zone, although in reality, because the moment of inertia of the solar convection zone is only about $10 \%$ of the moment of inertia of the radiative interior, it is the convection zone whose axis would be perturbed the most during any such adjustment. Nevertheless, the final state would be one in which the axis of the magnetic field in the radiative interior would be aligned roughly at the latitude of zero tachocline shear, which is also the latitude at which sunspots first appear at the beginning of a solar cycle. With respect to the axis of rotation, the magnetic field would no longer be axisymmetic, and would be rotating at the uniform rate of the radiative interior. I am assuming here that the final state is one of almost minimum kinetic energy, in which the entire Sun rotates about a common axis. 
The idea of there being an oblique magnetic solar rotator is not new. Dicke (1978), for example, postulated one for reconciling a variation in his surface oblateness measurements, thereby provoking a helioseismological discussion of evidence for its existence (Isaak, 1982; Gough, 1982). Mestel (2012) has provided a theoretical discussion of the pertinent dynamics.

At present it is impossible to predict the degree to which the nonaxisymmetric structure of the internal field would be imprinted on the field in the photosphere and beyond. However, one can seek observational guidance. It has been known for some time that there is a largescale approximately uniformly rotating magnetic asymmetry, now called the sector structure (Svalgaard and Wilcox, 1976), in the Sun's surface layers (Wilcox and Ness, 1965; Hudson, Svalgaard, and Hannah, 2014). It is generally believed that the uniformity of rotation is indicative of the dominant dynamics being imbedded deep inside the Sun, and associated with the solar dynamo presumed to operate mainly near the bottom of the convection zone or in the tachocline immediately beneath. Its variation has therefore been associated with either the Carrington rotation rate, the Bartels rate, or some similar indicator of surface variation (Svalgaard and Wilcox, 1976; Wilcox and Ness, 1965; Hudson, Svalgaard, and Hannah, 2014) with synodic period of about 27 days. Most analyses of the temporal variation of magnetic activity have that idea predominantly in mind. And indeed, because it is the turbulent motion in the convection zone that no doubt has the dominant influence on the surface magnetic field, this is a quite natural supposition. Moreover, there is observational evidence that under some circumstances large-scale structures do survive through highly turbulent flows: the persistence of the (terrestrial) Gulf Stream in the Atlantic Ocean (Stommel, 1958) and the von Kármán vortex streets that can be seen in atmospheric wakes behind islands ${ }^{1}$ are archetypal examples. It is therefore of interest to seek evidence for large-scale structure that is indicative of the rotation of the Sun's radiative interior. The crucial test is to establish that indeed the structure rotates more or less with the radiative interior, essentially maintaining rotational phase aside from small random variations brought about by the turbulent passage through the convection zone that even in the long term do not drift far from the essentially uniform rotation of the deep interior. Measurements of the solar-cycle variation in the kinetic energy and angular momentum of the convection zone (Komm et al., 2003; Antia, Chitre, and Gough, 2008) suggest that the corresponding short-term relative variation in the angular velocity of the radiative interior could be as great as $10^{-4}$; but the long-term variation must be very much smaller.

\section{Geomagnetic Evidence: A New Look at Old Data}

There appears to be no published direct statistical study of the Sun's sector structure addressing the long-term phase coherence that would be expected of the steady internal rotation. ${ }^{2}$ However, there is an interesting analysis of the geomagnetic field that could be pertinent (Svalgaard and Wilcox, 1975). A nonaxisymmetric rotating magnetic field, such as is associated with the sector structure, imparts a signature on the interplanetary field that in turn modulates the magnetic field surrounding the Earth. Svalgaard and Wilcox plotted - from 1926 to 1973 - a measure of the variation against time modulo a putative reference rotation period $\tau$ of 26.84 days synodic, and discussed mainly the dominant 'primary' component,

\footnotetext{
${ }^{1}$ http://www.eumetsat.int/website/home/lmages/lmage.

${ }^{2}$ de Toma, G. (2014), personal communication.
} 


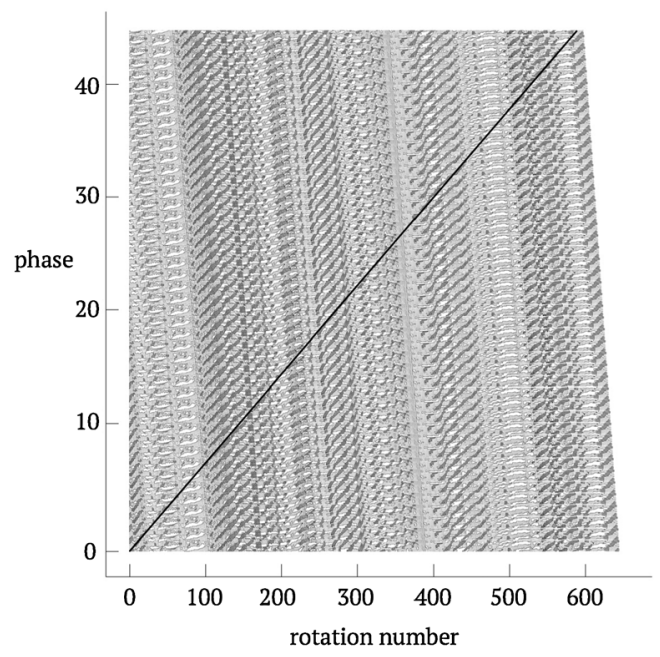

Figure 1 Representation of geomagnetic data throughout sunspot Cycles 16-20, constructed from Figure 5 of Svalgaard and Wilcox (1975). The original figure is essentially two vertical strips in each of which the abscissa is time modulo $\tau$, which here I call phase, and the ordinate, plotted downwards, can be regarded as rotation number (Svalgaard and Wilcox label it with calendar year). The two strips are identical aside from the shading used to indicate enhanced magnetic activity. The reference interval (period) $\tau$ is 26.84 days. The figure here has been constructed by taking just one of the original strips, in which the enhanced activity is either white or cross-hatched (the latter appearing dark grey in this greatly reduced rendition) rotated anti-clockwise by $90^{\circ}$, and stacking 44 identical copies above it, each having been displaced leftwards by $\tau$ to render phase continuous, and trimmed to make the left-hand edge of the data-containing region vertical. The abscissa is now the rotation number of the original (lowest) strip, from zero on 19 February 1926 to 645 in May 1973, and the ordinate is phase (in units of $\tau$ ). I draw attention to the dark grey regions that appear to trace straight lines inclined from the abscissa by 0.07025 periods/period. One such line is drawn in the plot. Because the features cling to the line, they appear to be a representation of an almost rigidly rotating magnetic feature that could be the signature of an oblique dipole in the radiative interior of the Sun, rotating at a rate $434.6 \mathrm{nHz}$ sidereal.

which appears to be closely associated with the chosen reference period, and is quite possibly a diagnostic of dynamo action principally in the equatorial regions of the convection zone, drifting in longitude as the sunspot cycle proceeds. But in addition, a secondary component rotating more slowly, with a period of about 28.5 days synodic, was identified. Does that component maintain its phase precisely? If so, is it an indicator of the oblique magnetic rotator in the radiative interior?

Figure 1 illustrates the geomagnetic variation. It was constructed from Figure 5 of Svalgaard and Wilcox (1975), which is composed of vertical strip pairs in each of which the abscissa (which here I call phase) is time modulo $\tau$, and the ordinate, increasing downwards, numbers the 645 rotation periods covered by the data. In the left strip of each pair, occurrences of enhanced magnetic intensity judged to be associated with the primary component are represented in solid black, and the secondary component is cross-hatched; the right strip simply reproduces the left strip, except that the primary component is represented in white, rendering the cross-hatching more noticeable. Figure 1 here is constructed from just the right-hand strips of Svalgaard and Wilcox's figure, placed end-to-end, 44 copies of which are appended laterally, each displaced upwards by $\tau$ to render phase continuous, and the whole rotated anti-clockwise by $90^{\circ}$. In addition, the left-hand edges of all but the original (rotated) strip (i.e., the lowest horizontal strip in Figure 1) have been trimmed so that the left-hand edge of the region containing data is vertical. The primary field varia- 
Figure 2 Seismologically determined rotation rate of the outer half of the solar interior obtained under the assumption that the entire Sun rotates about a common axis, plotted against fractional radius at various latitudes (taken from the US National Solar Observatory's archives). The horizontal dashed line denotes the cyclic frequency $434.6 \mathrm{nHz}$ deduced from the variation of the geomagnetic field.

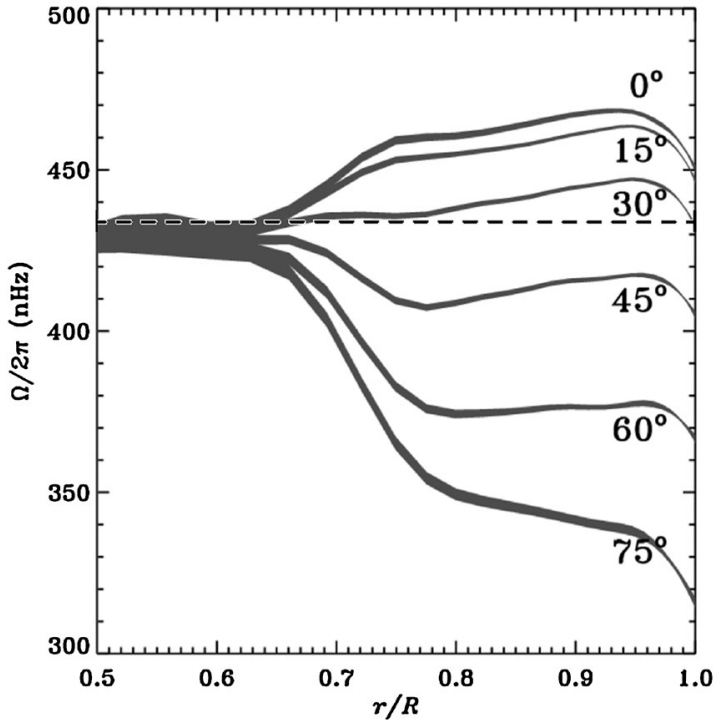

tion is evident as near-horizontal white stripes, not as prominent in this figure as are the black stripes in Svalgaard and Wilcox's original representation; the (weaker) cross-hatched regions are here more prominent, forwardly inclined from the primary component, indicating a temporal variation with lower frequency. I have emphasized an example of the latter with a straight line. The close fit of the data to that line appears to indicate that, aside from short-term deviations, long-term phase coherence may have been maintained over the entire interval of observation. The matter is not proven, however, because there were intervals near sunspot minima when there were insufficient reliable data for keeping track of phase. However, whenever the magnetic activity reappeared, it did so in phase with the earlier activity. The slope of the line in Figure 1, determined by uniformly weighted least-squares regression to the median phases of the cross-hatched features, is 0.07025 periods/period, which implies a mean recurrence period of those features of 28.73 days synodic (regression weighted by the feature duration yields the same period to the precision quoted here), corresponding to a sidereal cyclic frequency of $434.6 \mathrm{nHz}$. This coincides with the seismologically determined rotation rate $\Omega_{0} / 2 \pi$ of the Sun's radiative interior (Figure 2).

In addition to the linear regression depicted in Figure 1, separate straight-line leastsquares fits were made just in the intervals in which reliable data are available, namely for rotations $0-20$ (numbered from the beginning of the available data), 110-190, 230-310, $330-375,410-480,545-610$, and $630-644$. Subsequent regression against the midpoints of these regression lines yields a line $\mathcal{L}$ of slope of $0.07037 \pm 0.00018$ periods/period. This reproduces essentially the same sidereal cyclic frequency, $434.6 \pm 1.0 \mathrm{nHz}$, as that obtained by the single regression against the entire data set. The standard deviation $\sigma$ of the median phases from $\mathcal{L}$ of those subintervals is $0.161 \tau$, corresponding to about $60^{\circ}$ in longitude. This is less than the value $0.261 \tau$ that would be expected if the median phases of the seven regressions were random and uniformly distributed. Indeed, if the phases were uniformly random, the probability of the standard deviation being no greater than $\sigma$ would have been only 0.05 , which provides valuable, although admittedly not extremely strong, evidence for the magnetic rotator.

I conclude, therefore, that the 28.5-day feature identified by Svalgaard and Wilcox (1975) in the geomagnetic data may be evidence for there being an oblique magnetic dipole field 
in the Sun rotating with cyclic frequency $434.6 \pm 1.0 \mathrm{nHz}$ sidereal, the same rate as the helioseismologically determined rotation of the radiative interior. One might recall, however, that this is also the rotation rate of the photosphere at the latitude at which sunspots first appear at the beginning of each cycle, although I am aware of no alternative theory to relate the two.

Acknowledgements I thank Todd Hoeksema, Phil Scherrer, and Leif Svalgaard for interesting discussion, and Amanda Smith for helping with the preparation of the figures. I thank the referee for pointing out an ambiguity in the original caption to Figure 1, and for suggesting clarifying improvements.

Disclosure of Potential Conflicts of Interest The authors declare that they have no conflicts of interest.

Open Access This article is distributed under the terms of the Creative Commons Attribution 4.0 International License (http://creativecommons.org/licenses/by/4.0/), which permits unrestricted use, distribution, and reproduction in any medium, provided you give appropriate credit to the original author(s) and the source, provide a link to the Creative Commons license, and indicate if changes were made.

\section{References}

Antia, H.M., Chitre, S.M., Gough, D.O.: 2008, Temporal variations in the Sun's rotational kinetic energy. Astron. Astrophys. 477, 657. DOI.

Braithwaite, J., Spruit, H.C.: 2004, A fossil origin for the magnetic field in A stars and white dwarfs. Nature 431, 819. DOI.

Dicke, R.H.: 1978, The enigmatic solar distortion and the solar neutrino problem. In: Friedlander, G. (ed.) Status and Future of Solar Neutrino Research, 109.

Gough, D.O.: 1982, Evidence for an oblique magnetic solar rotator. Nature 298, 350. DOI.

Gough, D.O.: 2012, Pattern formation in rapidly oscillating peculiar A stars. Geophys. Astrophys. Fluid Dyn. 106, 429. DOI.

Gough, D.O., McIntyre, M.E.: 1998, Inevitability of a magnetic field in the Sun's radiative interior. Nature 394, 755. DOI.

Hudson, H.S., Svalgaard, L., Hannah, I.G.: 2014, Solar sector structure. Space Sci. Rev. 186, 17. DOI.

Isaak, G.R.: 1982, Is the Sun an oblique magnetic rotator? Nature 296, 130. DOI.

Komm, R., Howe, R., Durney, B.R., Hill, F.: 2003, Temporal variation of angular momentum in the solar convection zone. Astrophys. J. 586, 650. DOI.

Mestel, L.: 2012, Stellar Magnetism, 2nd edn., Oxford University Press, London

Schou, J., Antia, H.M., Basu, S., Bogart, R.S., Bush, R.I., Chitre, S.M., Christensen-Dalsgaard, J., di Mauro, M.P., Dziembowski, W.A., Eff-Darwich, A., Gough, D.O., Haber, D.A., Hoeksema, J.T., Howe, R., Korzennik, S.G., Kosovichev, A.G., Larsen, R.M., Pijpers, F.P., Scherrer, P.H., Sekii, T., Tarbell, T.D., Title, A.M., Thompson, M.J., Toomre, J.: 1998, Helioseismic studies of differential rotation in the solar envelope by the solar oscillations investigation using the Michelson Doppler Imager. Astrophys. J. 505, 390. DOI.

Stommel, H.: 1958, The Gulf Stream: A Physical and Dynamical Description, University of California Press, Berkeley.

Svalgaard, L., Wilcox, J.M.: 1975, Long-term evolution of solar sector structure. Solar Phys. 41, 461. DOI.

Svalgaard, L., Wilcox, J.M.: 1976, The Hale solar sector boundary. Solar Phys. 49, 177. DOI.

Wilcox, J.M., Ness, N.F.: 1965, Quasi-stationary corotating structure in the interplanetary medium. J. Geophys. Res. 70, 5793. DOI. 